\title{
Preoperative Assessment
}

\author{
Pawel Szychta1,2, Ken J. Stewart ${ }^{1}$ and Jan Rykala ${ }^{2}$ \\ ${ }^{1}$ Plastic and Reconstructive Surgery Department, St John's Hospital, Livingston \\ ${ }^{2}$ Plastic, Reconstructive and Aesthetic Surgery Department, $1^{\text {st }}$ University Hospital, Lodz \\ ${ }^{1}$ Great Britain \\ ${ }^{2}$ Poland
}

\section{Introduction}

Rhinoplasty offers a substantial customization of the parameters of the operated area in comparison with most cosmetic procedures. At the same time, the surgeon faces a challenging task of matching the complex shape of the nose to the rest of the face. The face is a three-dimensional structure of highly-integrated anatomical components, gently intersecting one another. Therefore, detailed preoperative planning, based on accurate knowledge of the construction of the nose, can significantly contribute to achieve pleasing result after rhinoplasty.

\section{Nasal aesthetics}

The nose occupies a central position on the face, dictating, to a large extent, general facial aesthetics. There is no single model of ideal proportions of the face, or nose. Moreover, a slight facial asymmetry is considered an attractive trait. In practice, therefore, the concept of the normal range should be used instead of determining the 'ideal' values of parameters describing the proportions of the face and nose.

The result of rhinoplasty should be an attractive nose, harmonious with the rest of the face and emphasizing the beauty of the eyes (Tardy, 1997). The most favourable evaluation of patient before rhinoplasty is based on the proportions of nose with the whole face.

\subsection{Nose as an integral part of the face}

Examination of the patient prior to rhinoplasty should include assessment of all the facial components as complementary elements. Knowledge of normal proportions allows for accurate detection of deviations from existing standards and precise targeting of surgical correction in the establishment of an aesthetic shape of the nose, which is proportionate to the rest of the face.

Leonardo da Vinci's facial model is split into three equal horizontal parts, bounded by the lines intersecting four topographic points: trichion (hairline in the midline), glabella, subnasale (nasal spine) and menton (lower edge of the chin) (Figure 1) (Gunter et al., 2007). The upper third is the least important in the estimation of the proportion of the nose and face. The nose is in the middle third of the face. The lower third of the face (between subnasale and menton) is further divided by a horizontal line intersecting the commissure of the lips (stomion) into two parts: $1 / 3$ upper and 2/3 lower. 
In addition, Powell and Humphreys divided face into 5 vertical areas of equal width, bounded by six vertical lines: both, a) and b) lines passing through the inner canthi, which includes the medial part of the face with nose, both, c) and d) lines crossing the lateral canthi, denoting the lateral edge of the neck, both, e) and f) lines through the most outwardly situated point of the pinna (Powell \& Humphreys, 1984).
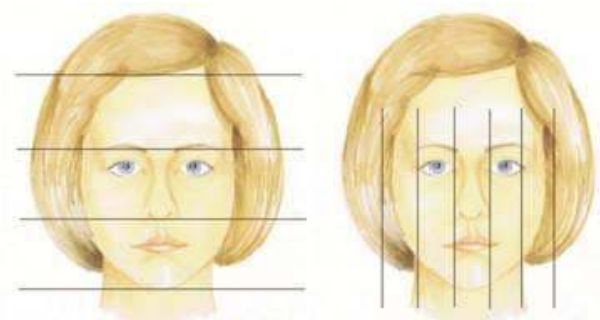

Fig. 1. a) Face divided into three equal horizontal parts by Leonardo da Vinci, b) face divided into 5 vertical areas of equal width

The correct proportions of the face vary depending on gender, race, and individual anatomical features. According to a beautiful face in relation to gender, attractive women have less marked jaw, bigger eyes and complementary smaller noses, as compared to men (Perrett et al., 1994). Aesthetically pleasing lips are fuller, with a smaller upper lip. Women also have a smaller distance between lips and chin. In contrast, men usually have a bigger nose than women, coupled with a deep placement of eyes, located close to each other. Attractive males have visible cheekbones and jaw. It is important that the ears in men are not prominent.

For educational purposes, the commonly used facial proportions relate to white women, who are the most common group of patients undergoing rhinoplasty (Talakoub \& Wesley, 2009). It should be noted, however, that surgeons have to maintain the different relationships between the nose and face in individuals of both sexes and different races.

\subsection{Nasal aesthetic subunits}

The facial surface is divided into so-called aesthetic units, which are areas of skin with identical properties such as colour, texture, elasticity and thickness, and are often limited by the curvature of the surface of the body. They serve as guidelines for reconstruction in the event of loss of tissue. One of the units is the skin covering the nose. Its surface is divided into subunits, the knowledge of which can be helpful in understanding the nasal shape. There are nine aesthetic subunits of the nose: tip, columella, alar bases, alar side walls, dorsum and dorsal side walls (Figure 2) (Rahman et al., 2010). Any surgical incision placed between the subunits produces the least visible scars.

\subsection{Characteristics of the correct shape of the nose}

Preoperative evaluation is based largely on a conversation with a patient, observation of the nose and palpation. However, standardised photographs are a valuable addition in the preoperative analysis of the nasal shape, often highlighting the anatomical details, which are difficult to see during the examination. Regardless of the method of analysis, the surgeon should recognize the anatomical abnormalities of the nose, based on knowledge of the 
correct proportions of the face and nose. The characteristics of the aesthetic face and nose are described below.

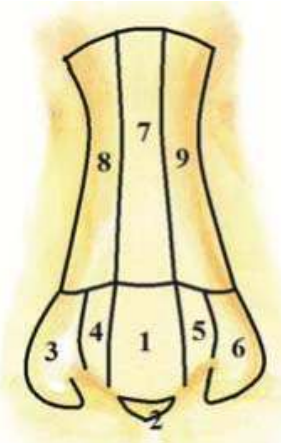

Fig. 2. Aesthetic subunits of the nose: 1 - tip subunit, 2 - columellar subunit, 3 and 6 - alar side wall subunits, 4 and 5 - alar base subunits, 7 - dorsal subunit, 8 and 9 - dorsal side wall subunits

From the anterior view (on examination/in the photograph), the nasal bridge changes gradually over its entire length, being narrowest in the area around the root (at the height of the medial supraorbital edge, nasion), while the broadest at the base (at the tip of the nose pronasale) (Krzeski, 2005). Lines running along its side edges should be slightly diverging caudally (Figure 3a). These are commonly described as the dorsal aesthetic lines.

Ideally, the nasal width at nasion should be equal to the palpebral fissure; while at the level of pronasale (width of the nasal base) should be similar to the distance between the inner commissures of the eyes. The width of the base of the nasal osseous pyramid should be 70$80 \%$ of the nasal base (Mathes \& Hentz, 2006).

The shape of the nasal base and columella should resemble the outline of seagull in flight (gull-wing appearance) (Figure 3b) (Gunter et al., 2007). Its shape is attributable to the columella position, which protrudes slightly below the edges of the nasal alae. The 'trunk of the gull' is the lowest portion of the columella, and the 'wings of the gull' are the outlines of the lower edges of alae. The edges of alae are shaped like a dome, convex in caudal-lateral direction.

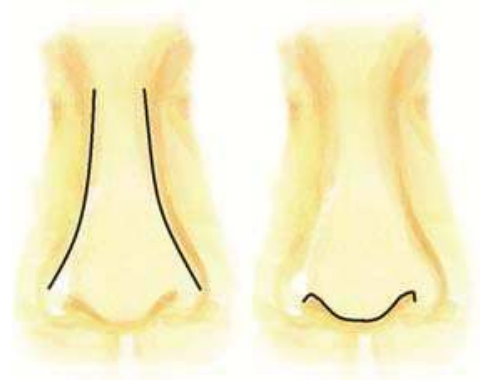

Fig. 3. a) Outline of the edges of the nasal bridge b) gull-wing appearance of the nasal base and columella 
In addition, the proportions between the nose and mouth should be considered. The length of the columella should be equal to height of the upper lip (Simons, 1982). Similarly, the upper lip height (distance between subnasale and labiale superius) should be equal to 1/3 of the lower face ( $1 / 9$ of the whole face, according to the Leonardo da Vinci's canon of beauty) (Trenite, 2005). From the profile, the alar edge should have the shape of the letter 'S', starting at the front of the columella, and ending posteriorly and laterally at the transition between the nasal ala and cheek (Figure 4) (Tardy, 1997).

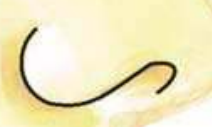

Fig. 4. S-shaped alar edge of the nose

The most attractive nasal profile is straight, as compared to convex or concave. The nasal length and the columella should be proportional, without local irregularities. The nasal profile is well described by the frontonasal angle. The frontonasal angle starts at around eyebrows area and creates a gentle concave arc ending at the bridge of the nose. The top of the frontonasal angle is positioned at level between the free edges of eyelids and supratarsal folds, with eyes opened freely. In women, the nasal profile is straight or slightly concave, in males straight or slightly convex (Tezel \& Durmus, 2009).

The nasal tip has two points that define it in the horizontal plane, which are the points on the domes of the nasal alae. Another two points, defining the tip in the profile view, are supratip and columellar breakpoints. Supratip breakpoint is the the stepoff from the the plane of the nasal dorsum on profile view at the cephalic aspect of the nasal tip. Columellar breakpoint forms about $2 \mathrm{~mm}$ anterior part of the columella (Figure $5 \mathrm{a}$ ). All four points that define the nasal tip form two equilateral triangles (Figure 5 b) (Daniel, 2009).

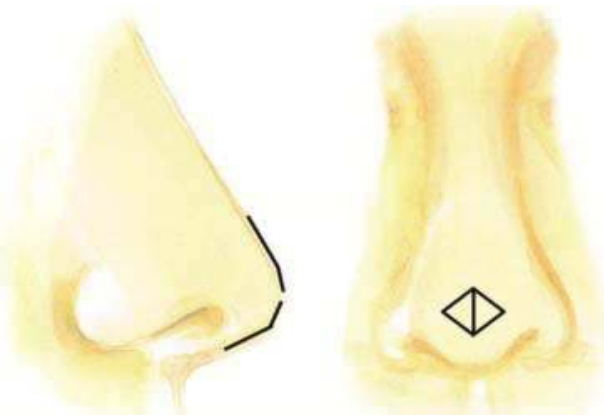

Fig. 5. a) Points defining the nasal tip in the vertical plane, visible from the profile; b) Two equilateral triangles formed by the points defining the nasal tip and the domes of the nasal alae 
Projection of the nasal tip is determined by the distance from the junction of the upper lip with alae (subnasale) to the most anterior edge of the nose (pronasale) (Elsahy, 2000). Given the normal projection of the upper lip, $50-60 \%$ of this line is located anterior from the most prominent point of the upper lip (Mathes \& Hentz, 2006).

Recession of the supratip area is beneficial for women, but does not occur commonly in men. Nasal tip shows more cranial rotation in women (described as supratip break) and therefore is more apparent in females, in contrast to the nasal bridge, which is more prominent in men (Begg \& Harkness, 1995).

The worm eye view shows the nasal base. Its external outline in the ideal conditions should create an equilateral triangle (Figure 6). The height of the triangle, measured from the nasal tip (pronasale) to the back of the nose (subnasale) consists of three parts. Its anterior $1 / 3$ passes only through the infratip lobule, whilst the posterior two thirds are located along the columella and nostrils. In another division, its length is divided into two halves at the division of the medial edge of nostrils from the columella. The nostrils have the shape of falling drops, and their widths are similar to the width of the columella. The long axis of the nostrils faces anteriorly and medially about 45 degrees in comparison to the axis of the columella. The width of the columella is narrow in its central part, and widens posteriorly, which reflects the anatomy of the medial crura of alar cartilages. Anteriorly the columella changes into the infratip lobule, whilst posteriorly it connects to the upper lip (Elsahy, 2000).

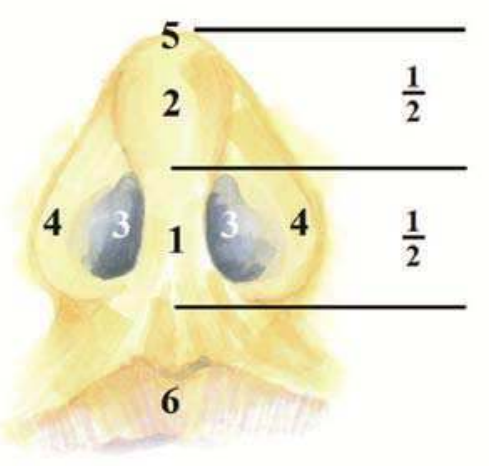

Fig. 6. Base of the nose; 1 - columella, 2 - infratip lobule, 3 - nostrils, 4 - alae, 5 - nasal tip, 6 - upper lip

\subsection{Anthropometric measurements of the face and nose}

The shape of the cartilaginous-osseous skeleton is relatively well depicted on the skin surface due to the closely fitting skin envelope, especially in patients with thin skin. Therefore, it is possible to designate relatively fixed points of surgical anatomy, which are used for preoperative and intraoperative evaluation. After determining points on the face, anthropometric parameters can be taken, which consist of linear measurements with the resulting indicators, angles and spatial parameters (Szychta et al., 2010).

On the face we suggest determination of 24 anthropometrical points (7 separate for left - L and right - $\mathrm{R}$ sides), including points describing the nostrils: anterior (naL, naR), posterior $(\mathrm{npL}, \mathrm{npR})$, lateral (nlL, nlR) and medial (nmL, nmR), as well as: subalare (sbalL, sbalR), 
alare (alL, alR), alar curvature point (acL, acR). Nine single points are also determined: pronasale (prn), infratip lobule (il), subnasale (sn), nasion (n), glabella (gl), labiale superius (ls), rhinion (rh), columellar point (cp), stomion (s) and menton (m) (Figure 7).
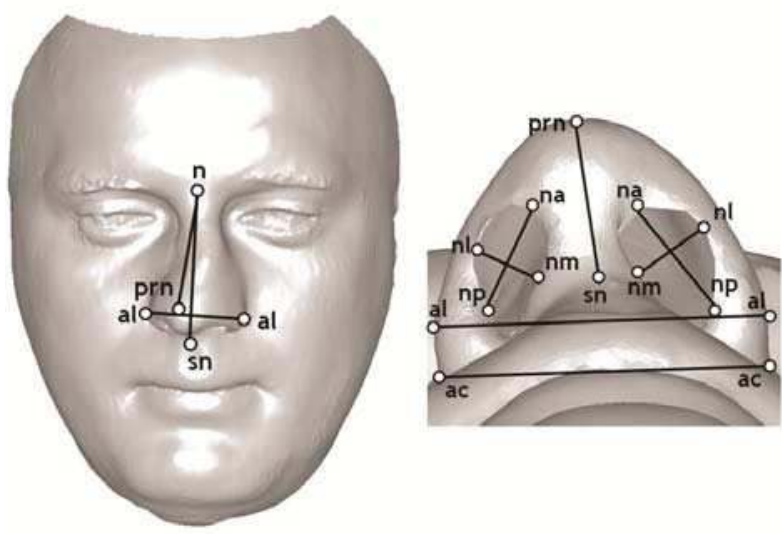

Fig. 7. Anatomical anthropometric points of the face shown on the three-dimensional model of the face; views: a) from the front, b) from the bottom

The nasion (n) is defined in the place of the greatest concavity of the upper pole of the nasal bridge (nasal root). The rhinion ( $\mathrm{rh}$ ) is located at transition of cartilage into osseous pyramid. The pronasale (prn) is described as the most projecting point at the nasal profile. The subnasale (sn) is defined by the base of columella. The subalare (sbal) is located at transition of the ala into the inferior wall of nostril. The alar curvature point (ac) is located in the far lateral basal part of the ala. The alare (al) is the most laterally situated point of the nose. The columellar point (cp) is determined at the intersection of both axes of nostrils, usually being the lowest point of the nasal tip. The stomion (s) is located at the level of the lips fissure, whilst the menton $(\mathrm{m})$ is the lowest point of the chin. The reliability of measurements using the anatomical points has been confirmed in the previous studies. The above mentioned anthropometric points can be used to obtain: 9 linear measurements, 7 indicators of nasal proportions, 7 angles, 2 indicators of spatial asymmetry of the skin surface and the total volume of the operated area.

The linear measurements include: length and width of both nostrils (naR-npR, naL-npL, nlR-nmR and nlL-nmL, respectively), nasal height (n-sn), nasal length (n-prn), nasal width (alL-alR), length of the nasal base (acL-acR) and the nasal prominence (sn-prn). Nasal length (n-prn) should be equal to the distance between stomion and menton (s-m). Nasal height is equal to the length of the upper lip or up to $50 \%$ longer. Nasal prominence (sn-prn) is about $2 / 3$ of the nasal length (n-prn). The degree of projection of the nasal tip, determined by the distance sn-prn, should be equal to the upper lip height.

The calculated linear indices are: the index of the nasal base (sn-prn/ac-ac* 100), the index of the prominence to the nasal width (sn-prn/al-al * 100), indicators of the shape of both nostrils (nl-nm/na-np * 100), nasal index (al-al/n-sn * 100) and index of the nasal length (nprn/n-sn * 100). Asymmetry in the shape of the nostrils is given by [2* ((nlL-nmL/naL-npL) - (nlR-nmR/naR-npR)) / ((nlL-nmL/naL-npL) + (nlR-nmR/naR-npR)) * 100]. The nasal index is normally $55 \%-60 \%$. 
The analysed angles are: interaxial angle (sbalR-cp-sbalL), angle of deviation between cutaneous septum and midline (cp-sn-median line), angle of deviation between osseous pyramid and midline (rh-n-median line), angle of deviation between osseous and cartilaginous pyramid (n-rh-prn), nasofrontal (gl-n-prn), nasolabial (cp-sn-ls) and nasofacial (n-prn-sn) (Figure 8).

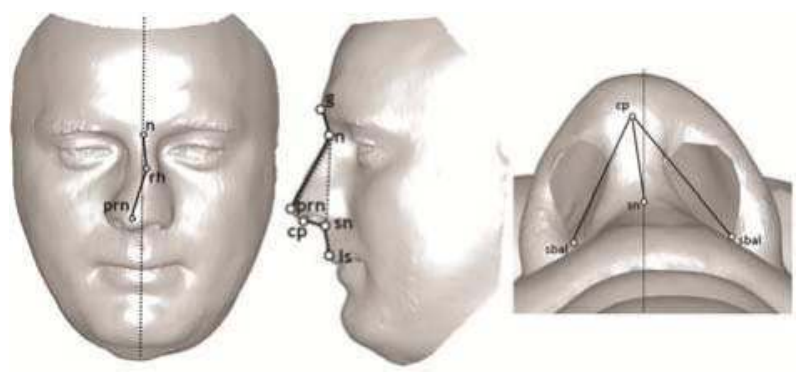

Fig. 8. Angles of the nose, shown on the three-dimensional model of the face; views: a) from the front, b) lateral profile, c) from the bottom

The nasofrontal angle is normally 125-135 degrees. However, the following features of the nasofrontal angle are important for the whole aesthetics of the nose, apart from its value: position of its tip in the frontal and sagittal plane, and its slope. In fact, the nasofrontal angle does not contain a clearly identified vertex, but describes a mild change in the nasal profile. The nasolabial angle in women is 95-105 degrees, while in men it varies from 90 to 95 degrees (Gruber \& Peck, 1993). The nasolabial angle directly sets the rotation of the nose. It may be different from the columella-lip angle, e.g. the prominent edge of the caudal nasal septum can cause an illusion of increased cranial rotation of the nose, although the nasolabial angle may remain within normal limits. The nasofacial angle, determining the slope of the nasal bridge of the nose, sets the deviation of the nasal bridge from the facial plane and varies correctly from 34 to 35 degrees (Daniel \& Farkas, 1988). An excessively obtuse nasofacial angle indicates excessive projection of the nose, and a very sharp nasofacial angle is often found e.g. in boxers.

Spatial measurements are only possible by using three-dimensional imaging, which has increasing clinical application. Asymmetry of the side surfaces of the nose is described as: [2 * (left side skin surface area - area right) / (area left + area right) * 100], while the asymmetry of cross-sectional areas of the nostrils is equal to [2* (left cross-section area -area right) / (area left + area right $)$ * 100]. Automated measurement of the total volume of the nose can also be performed (Szychta et al., 2010).

Certainly not all patients will require undergoing the whole abovementioned extensive formal analysis. Such exhaustive measurements are usually important in revision or cosmetic cases with a high degree of precision required. That said, it is important for surgeons to have a working understanding of all of these parameters.

\section{The functionality of the nose}

\subsection{The nasal valve}

Internal nasal valve is defined as that area of maximal narrowing that is bounded by the nasal septum, caudal aspect of the upper lateral cartilage, the anterior face of the inferior 
turvinate and the nasal floor. It is located at a distance of about $1.3 \mathrm{~cm}$ posterior to the nostrils (Trenite, 2005). A narrowed nasal valve is the most common cause of reduced patency of the nasal airway, caused by the distortion of the nasal anatomy. Air resistance in the nasal passages may also be caused by incorrect construction of the nasal vestibule or the pathology of the nasal cavity valve.

The external nasal valve, formed by the nasal vestibule caudal to the internal nasal valve, is defined by the alar and lower lateral cartilage tissues, which create the lateral and anterior walls, as well as by the caudal septum and piriform aperture.

Cottle's test is used to detect internal nasal valve pathology (Trenite, 2005). The surgeon places his hand on the patient's cheek near the nasal bridge and then pulls the skin in the lateral direction. In case of valve failure, the patient feels immediate relief on breathing after previous breathing difficulties (positive result). A negative result usually indicates either lack of any pathology in the absence of nasal resistance or a correct nasal valve with pathology of the other area of the nose. A false positive result is found in case of nasal alae collapse. False negative results occur in cases of stenosis or adhesions of the valve or medial displacement of the frontal process of maxilla as a result of mechanical trauma or surgery (Krzeski, 2005).

Another way to evaluate the nasal valve is the introduction of the blunt instrument (e.g. speculum) to the vestibule, moving away the nasal side wall from the septum. Improved function of the nose on inspiration demonstrates pathology of the valve (Krzeski, 2005). Nasal valve collapse is corrected with cartilage grafts. Nasoscopy and cross-sectional imaging have value in illustrating the cause of airway obstruction.

\subsection{The mechanism supporting the nose}

The cartilaginous-osseous skeleton provides the nasal scaffolding and supports the covering soft tissue (Figure 9). Understanding the mechanisms supporting the nose contributes to an optimal range of surgical manipulation, including the ability to predict correctly the postoperative appearance of the nose after wound healing.

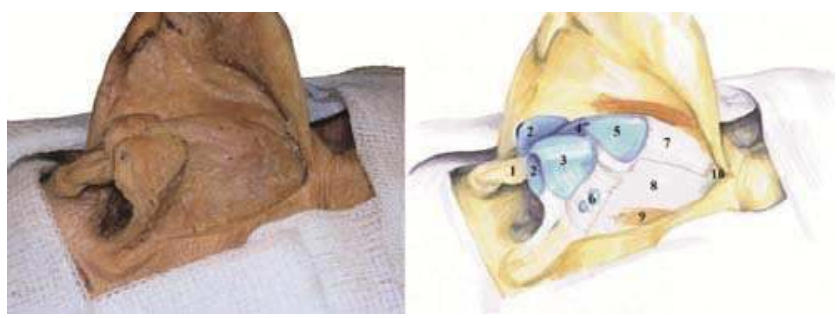

Fig. 9. The cartilaginous-osseous skeleton acting as a support mechanism of the nose: a) cadaveric dissection $b$ ) scheme depicting the anatomical details: 1 - skin septum, 2 - medial crus of nasal ala, 3 - lateral crus of nasal ala, 4 - nasal septum, 5 - upper lateral cartilage, 6 pisiform cartilage, 7 - nasal bone, 8 - frontal process of maxilla, 9 - nasal muscle, 10 - nasal part of frontal bone

There are several mechanisms to support the nose with different significance for individual patients. However, there are three relatively stable mechanisms, which have the greatest impact on the stability of the nasal tip: a) Medial and lateral alar crura (size, shape and structure), b) Ligamentous attachment between the upper lateral nasal cartilages and the 
alae, and c) Connections between the medial crura of alae and the septum. The less important six elements supporting the nose include: a) ligamentous connection of the alar domes, b) the nasal septum, c) the pisiform cartilages, extending a support function of the lateral crura of alae to the pyriform aperture, $d$ ) adhesions of the alae to the covering muscle and skin, e) the nasal spine and f), the membranous septum (Figure 9) (Tardy, 1997). It should be noted that the classic three major support mechanisms are not always the major contributors in all patients. For example, in patients with a tension nose, the nasal septum usurps much of the supporting role of the tip, and may be the single most important support mechanism.

The mechanism for support for the nose can be assessed by checking the 'reversing mechanism'. The nasal tip is squeezed by the thumb and after a rapid withdrawal of the finger the tissue is observed to return to baseline. A slow or incomplete return to the original shape may indicate a weak support apparatus.

Operations, which maintain the structural integrity, result in controlled postoperative outcome. A skeleton of sufficient strength maintains its functional support of the skin, subcutaneous tissue, and SMAS. Moreover, larger noses after subtle correction may be more aesthetically pleasing than small noses after radical reduction. Favourable outcome of nasal correction can be achieved in most cases with conservative reorientation of the anatomical elements.

\section{Nasal deformities}

\subsection{Deviation of the nasal septum}

Deviation of the nasal septum should be assessed in the context of the whole nasal skeleton (Figure 10). It often coexists with distortion of the nasal pyramid. The surgeon assesses the visible distortion and examines for fractures using the speculum.

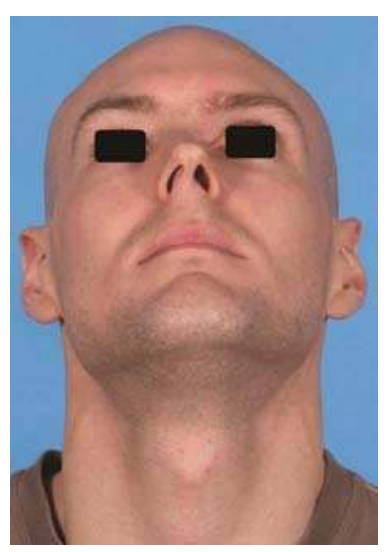

Fig. 10. Twisted nasal septum

\subsection{Distortion of the nasal pyramid}

Distortion of the nasal pyramid, resulting in impaired function, is a frequent clinical problem, both functionally and aesthetically. In case of deformation of the nasal skeleton, the primary aim is improved breathing. A second aim is correction of nasal aesthetics. A 
crooked nose is often difficult to repair due to the distorted and scarred skeletal elements and necessitates bilateral surgical manoeuvres to restore symmetry. The importance of the nasal septum in the pathogenesis and the subsequent persistence of crooked nose deformity after surgery should be emphasized. The septum is much like the rudder of a boat. If it is deviated and not corrected, it will steer the nose back to a crooked position even after corrective osteotomies are performed.

\begin{tabular}{|c|c|c|c|}
\hline $\begin{array}{c}\text { Diagnosed type of } \\
\text { distortion of nasal } \\
\text { pyramid }\end{array}$ & $\begin{array}{c}\text { Distortion of the cartilaginous- } \\
\text { osseous skeleton in relation to } \\
\text { the median facial line }\end{array}$ & $\begin{array}{c}\text { Shape of } \\
\text { the nasal } \\
\text { bridge }\end{array}$ & $\begin{array}{c}\text { Position/s of the } \\
\text { nasal bridge } \\
\text { bend/s }\end{array}$ \\
\hline Oblique nose & $\begin{array}{c}\text { The cartilaginous and osseous } \\
\text { arch veers in the same direction }\end{array}$ & $\begin{array}{c}\text { Straight } \\
\text { line }\end{array}$ & Nasion \\
\hline $\begin{array}{c}\text { Distortion of the } \\
\text { cartilaginous } \\
\text { pyramid }\end{array}$ & $\begin{array}{c}\text { The osseous pyramid is } \\
\text { straight, the cartilaginous } \\
\text { pyramid distorted laterally }\end{array}$ & $\begin{array}{c}\text { Singly } \\
\text { twisted } \\
\text { curve }\end{array}$ & Rhinion \\
\hline $\begin{array}{c}\text { C-shaped distortion } \\
\text { of the nose }\end{array}$ & $\begin{array}{c}\text { The cartilaginous vault is } \\
\text { shifted in the opposite direction } \\
\text { to the osseous pyramid }\end{array}$ & Arch & $\begin{array}{c}\text { Rhinion and } \\
\text { nasion }\end{array}$ \\
\hline $\begin{array}{c}\text { S-shaped distortion } \\
\text { of the nose }\end{array}$ & $\begin{array}{c}\text { The cartilaginous vault shifted } \\
\text { in the opposite direction to the } \\
\text { osseous pyramid }\end{array}$ & $\begin{array}{c}\text { Double- } \\
\text { twisted } \\
\text { curve }\end{array}$ & $\begin{array}{c}\text { Nasion, rhinion } \\
\text { and area of } \\
\text { weakened } \\
\text { triangle }\end{array}$ \\
\hline
\end{tabular}

Table 1. Division of nasal pyramid deformation, depending on the distortion of the nasal bridge from the midline

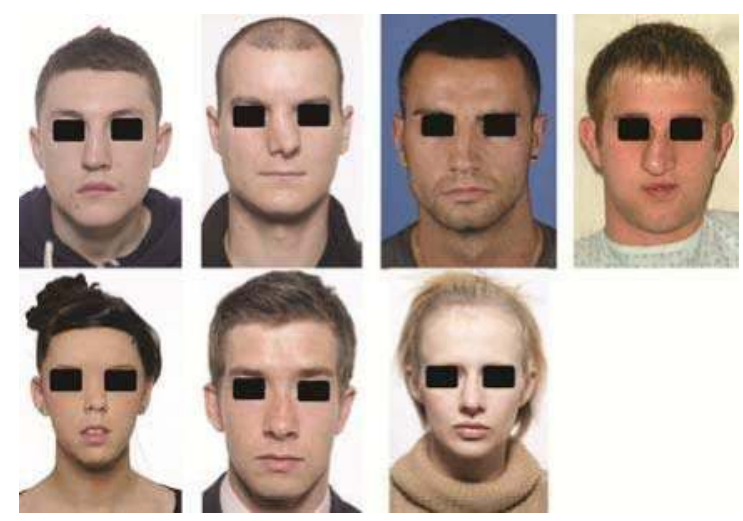

Fig. 11. Division of nasal pyramid deformation, depending on the distortion of the nasal bridge from the midline; a) oblique nose, b) nose with distorted cartilaginous vault, c) ' $C^{\prime}$ shaped nose, d) 'S'-shaped nose

Post-traumatic deformity reflects the type and direction of injury. In terms of the extent of cartilaginous and/or osseous damage this may include: a) distortion of the nasal cartilaginous vault by a deviated nasal septum, b) distortion of the entire cartilaginousosseous skeleton, or c) displacement of the osseous nasal pyramid. Distortion of the external 
nose, depending on the type of deviation of the nasal bridge from the midline, is described as: a) an oblique nose, b) a hooked nose, c) a saddle nose, or d) and e) ' $C$ ' or ' $S$ ' shaped nose (Table 1, Figure 11) (Krzeski, 2004). Assessment of the extent of injury helps in planning the appropriate surgical technique. Preoperative evaluation and the subsequent surgical correction should consider separately the three parts of the nose: cranial, middle, caudal.

\subsection{The hooked nose}

The nasal bridge is built of bone and cartilage. The osseous part is formed by the paired nasal bones, and the cartilaginous is built of lateral nasal cartilages attached to the nasal septum. Their correct proportions define the aesthetics of the nasal bridge. In women this is a straight or slightly concave, whilst in men forms a straight or gently curved line. The nasal hump is an excessive bulge which creates a hooked appearance (Figure 12). The aim of resection of the hump is aesthetic correction that will reconstruct the optimal nasal proportions (Sevin A et al., 2006).

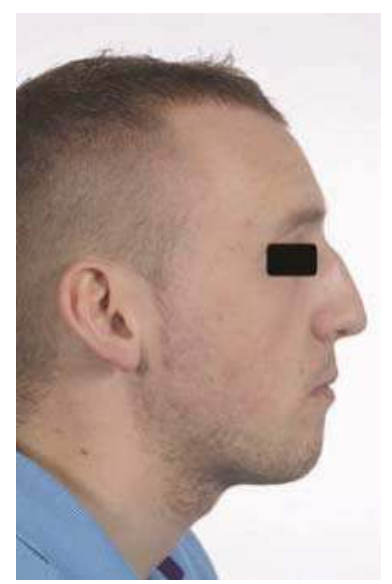

Fig. 12. Hooked nose (nasal hump)

Various anatomical structures may contribute to the nasal hump. These include the osseous pyramid and the cartilaginous vault. However, a genuinely pronounced nose may also give the illusion of a hump. Alleged hump (also called pseudo hump, usually resembling a hump of the osseous pyramid) results from the reduced projection of the upper cartilaginous vault or ptotic tip, giving the impression of excessive convexity of the caudal edge of the nasal bones. The prominent nose includes hypertrophy of the septum cartilage in the sagittal plane and the accompanying hypertrophy of the lateral cartilages of the nose (Krzeski, 2004).

\subsection{Saddle nose}

The saddle nose is determined by collapse of the nasal bridge, often colloquially described as a 'boxer's nose' or 'puggy nose' (Figure 13).

The saddle nose can be congenital but it is more often acquired. Aetiologies include: iatrogenic cause, certain types of cleft lip and palate, genetic syndromes, congenital syphilis, recurrent cartilaginous inflammation, Wegener's granulomatosis, cocaine use or leprosy. 

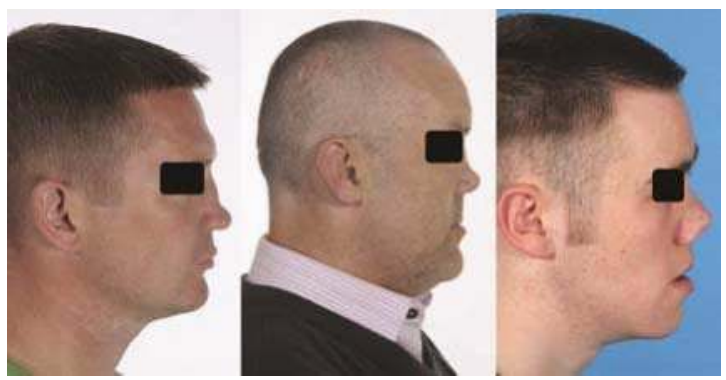

Fig. 13. Saddle nose

A practical method of classification described by Tardy divides severity of the saddle nose deformity into three categories:

a. Minimal - recession of tip supratip area is larger than the ideal 1-2mm, no retraction of the columella, the nose is not widened.

b. Moderate - loss of the nasal projection is mostly due to the damage to nasal septum cartilage. Damage to the cartilage of the nasal septum is a direct cause of weakening of two of the support mechanisms of the nose: the attachment of the medial alar crura to the caudal edge of the septal cartilage and the upper lateral cartilage attachment to the nasal alae. This is the reason for the reduced projection of the nose. The columella is retracted. The pyramid of the nose is too broad and flattened. The nasolabial angle is usually less than 90 degrees. In contrast, the nasal valve angle is often found increased, in rare cases up to 90 degrees.

c. Significant - significant loss of cartilage and loss of projection of the nasal bridge. In cases of a high degree of distortion, the following coexisting distortions are found: a lowered nasal bridge, a shortened columella, loss of support of the nasal tip, shortened nasal length, excessive cranial rotation of the nasal tip, widened nasal tip, alae too prominent and slender, atrophy of the nasal spine and the caudal edge of nasal septum. A decreased nasal tip projection together with a shortened columella is the cause of a disproportionately widened nasal base (Tardy, 1997).

\subsection{Distorted nasal tip}

The nasal tip is the most prominent area of the nose. Distorted proportions significantly aesthetically influence the facial aesthetics. A larger nose, harmonious with the face is often regarded as more acceptable than the insufficiently prominent one.
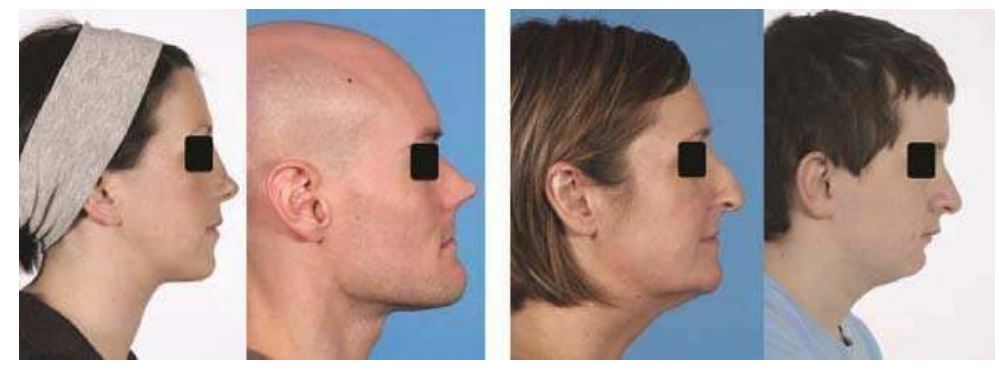

Fig. 14. Rotated nasal tip in women and men; a) snub nose b) droopy nose 
In addition to the position, the tip is also determined by caudal or cranial rotation. Excessive cranial rotation, providing a 'snub nose' is acceptable for women and unacceptable in men (Figure 14). On the contrary, 'hanging nose', caused by excessive caudal rotation, is more acceptable in men (Begg \& Harkness, 1995). It is often referred to in women as a 'witch nose'.

The supratip area is located between alae and the lateral cartilages. It is filled with adipose tissue. Insufficient volume of this structure can contribute to increased cranial rotation.

\section{Consultation of the patient}

Rhinoplasty is performed to obtain a functional nose of the correct proportions. Surgeons should acquire the ability to assess the executable range of corrections of the nose, having imposed the pre-established limits. These include anatomical features, such as the characteristics of the covering skin (thickness, elasticity, existing scar tissue), and the cartilaginous-osseous skeleton. In addition, the surgeon should note the areas of the wellproportioned nose, avoiding the changes during the operation (Tardy, 1997).

\subsection{Taking a history}

On the first visit, the surgeon evaluates the preoperative indication for rhinoplasty, faces the patient's expectations and confronts them with limited operational capability because of the individual anatomical conditions. In the meantime, the surgeon performs the initial analysis of the face and its proportions.

\subsubsection{Assessment of patient's motivation}

It is essential that the patient himself/herself is convinced of the need for surgery, without pressure from third parties. Recent traumatic experiences in the patient's private or professional life should be regarded as contraindications to surgery. Any signs of body dysmorphic disorder should be also identified. Patient with body dysmorphic disorder focuses usually on one part of the body, most commonly on the nose. His/her preoccupation with an imagined defect in the physical appearance often is expressed as require for surgical correction. The aesthetic outcome of the rhinoplasty is usually not satisfying for these patients with underlying psychiatric disorder (Alavi et al., 2011). The patient qualified for rhinoplasty should be emotionally mature, have realistic expectations about correction and understand his/her contribution to the healing process. With the above conditions of selection, the patients in most cases are happy with a good postoperative result.

By contrast, patients who survived a major injury to the nose are a distinct group of people. For the most patients, they never have considered undergoing surgery without the emergence of posttraumatic deformities to the nose and the related disorder of nasal patency. They want to breathe without difficulty and to have the shape of their nose reconstructed from period before injury. Their motivation is high and usually results in a positive reaction to rhinoplasty (Dziewulski et al., 1995).

The surgeon should be able to identify patients who may pose significant potential problems. These include patients: with unrealistic expectations, hyperaesthetic, obsessivecompulsive, depressive, fickle, hesitant, rude, overly flattering, chummy, sloppy, noncooperating, 'VIP', overly talkative or haggling over the price of treatment (Tardy, 1997). It 
should be emphasized that most patients have realistic expectations and understand limited possibilities of correction.

\subsubsection{Taking a history - nose}

At the beginning of the interview, the surgeon obtains information on conditions that may interfere with nasal function, taking into account: the patency of both nostrils, nasal trauma, seasonal allergies causing nasal obstruction, purulent sinusitis requiring antibiotics, snoring, epistaxis, asthma, headache caused by sinus, bronchitis, history of rhinoplasty or septoplasty, hypertension, tendency to keloids, drugs (aspirin, steroid sprays, decongestants), environmental conditions - work, addictions: cigarettes, alcohol and cocaine (Krzeski, 2004). There is potential of exacerbation of some of these diseases after the surgery. The above mentioned drugs should be discontinued at least one week prior to surgical treatment. Stimulants significantly impair wound healing and can lead to increased incidence of complications. Allergic rhinitis, causing obstruction of the nose, may be due to the hypertrophic inferior nasal turbinates. On this account, the history of headache should not be ignored, as it can exist due to insufficient warming of air inhaled by the inferior nasal turbinates.

Secondly, the surgeon asks questions about patient's concerns in relation to nasal deformations, allowing him/her to answer freely in order to understand the expectations and to assess of executable potential of the requests.

Preliminary analysis of the major complaints gradually shall turn into an assessment of less significant problems. Sometimes patients can not accurately identify problems. Then the surgeon acts as a guide, helping the patient for self-analysis of irregularities by using his experience and artistic sense. Useful descriptors include the words 'harmony' and 'proportion'. To better explain the operative plan to the patient, the surgeon demonstrates certain existing distortions of the individual nose, such as asymmetry, deformities of the nasal tip, distortion of the supratip area, irregularities of the nasal pyramid and nasal septal perforations. It is emphasized that rhinoplasty does not improve the facial symmetry. Sometimes it may become even more apparent after creating a symmetrical nose (Figure 15).

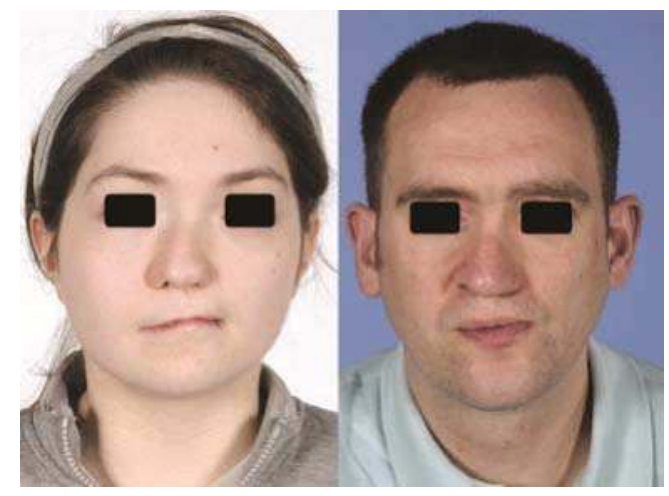

Fig. 15. Asymmetrical faces where rhinoplasty is contraindicated

The first visit should be completed by a thorough understanding between the surgeon and the patient of the aesthetic concerns and opportunities. It is followed by depiction of the 
operational plan, based on the realistic possibilities of correction of the distorted anatomical components, but also maintaining some of the existing pre-operative characteristics of a desirable nasal shape. In patients without respiratory distress, the surgeon should have in mind the need to maintain the functionality of the nose after surgery.

\subsubsection{Preoperative information}

Most patients are highly motivated and, after giving the relevant information, do not require a second preoperative interview. Before being asked for the formal consent, they should be informed about possible complications: bleeding, infection, distortion of results from the negative healing, the potential need for reoperation, which may not be possible until 1 year after the first procedure. However, the surgeon should reassure patients that rhinoplasty is usually a relatively safe operation, and that some minor irregularities may disappear in the process of healing.

In the event of the patient deciding to proceed, the surgeon should obtain written consent and photographic documentation. The patient receives a written recommendation concerning the necessary laboratory tests and guidance prior to surgery. Patients often want information about anaesthesia, operative time, length of hospital stay and early postoperative period. They should be informed about the possibility of postoperative pain, swelling of the face, bruising around the eyelids, difficulty in breathing through the nose due to anterior packing and the dressing left for up to seven days. In the case of the planned osteotomy patients should be warned about the need to swap glasses for contact lenses for 6 weeks after surgery.

\subsection{The physical examination of the external nose}

The surgeon examines the external nose and nasal cavity. At the beginning, an overall evaluation of nasofacial harmony is conducted. Examination of the external nose includes observation and palpation. Firstly, the skin covering the nose is assessed. Then, a systematic analysis of the nose is performed, including the following areas in the caudal direction: the nasofrontal angle, osseous pyramid, the upper lateral cartilage and nasal tip (the supratip area, nasal tip, alae, columella).

\subsubsection{Evaluation of nasal skin and subcutaneous tissue}

The surgeon can relatively easily modify the nasal cartilage and bone, but the nature of the covering skin must be accepted and included in the technique used for correction. Individual characteristics of each patient's skin have a huge impact on the scope of executable operations and the final result. Nasal skin is resistant to varying degrees to changes in cartilaginous-osseous skeletal, depending on its thickness, texture and elasticity. The elasticity of the skin is reduced in the elderly. The constraints due to the volume of preoperative skin with reduced elasticity must be taken into account during preoperative planning. For example, the skin over a broad nasal base may not contract adequately to cover a small, narrow base of the nose, and the postoperative result rather turns into a distorted wide base of the nose.

The skin is assessed by observation and palpation, checking the extent of the possible surgical correction. Gently lifting the nasal skin allows for an evaluation of its mobility in relation to the nasal skeleton and for the indirect analysis of its elasticity and contractility. 
The thickness of the skin is the single most important parameter of the skin. Nasal skin is generally thinner, more mobile, easier shifting in the cranial area, while being relatively devoid of subcutaneous tissue and sebaceous glands. The caudal part of the nose contains a thicker skin more strongly attached to the substrate, with a higher content of the sebaceous glands. Skin is thickest in the following areas: the nasal root (nasion), supratip area, whiles the thinnest at the rhinion (Krzeski, 2005). This should be taken into account when considering nasal reduction. Nasal tip contains additional subcutaneous tissue.

The best skin for the perfect result of rhinoplasty is of moderate thickness. It consists of the epidermis that includes minimum number of sebaceous glands and pores. Sufficient quantity of fibrous tissue and fat protects the skin from underlying skeletal structures, while hiding minor irregularities in the nasal cartilages. It adheres well to the bed after the surgery, so that changes made to the skeleton translate into the desired changes in the appearance of soft tissue.

In patients with thin, delicate skin, less postoperative swelling and fast wound healing are observed (Figure 16 a). However, it does not disguise small irregularities in the skeleton, making the shape of the nose edgy and unnatural. In most patients, the skin is medium thick or thin, so the surgeon should make every effort to save the subcutaneous tissue during operation (Tardy, 1997).

Thicker nasal skin is un-aesthetic (Figure 16 b). For those with thicker skin, a higher postoperative swelling is observed, together with slower healing and more pronounced contraction. The creation of scar tissue under the skin is more pronounced, especially for a nasal reduction. This leads to a parrot nose (pollybeak), a shapeless nose. It is difficult to obtain the accentuated definition of the nose in patients with thick nasal skin. After the operation is adheres poorly to the nasal skeleton. Care should also be taken not to resect excessively the cartilaginous skeleton due to the danger of weakening the support of the nose (Mathes \& Hentz, 2006).

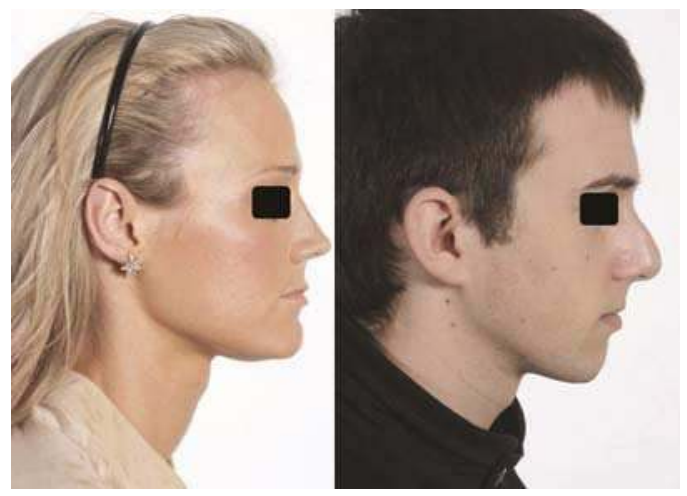

Fig. 16. Noses with a) thin, b) thick nasal skin

\subsubsection{Examination of the cartilaginous-osseous nasal skeleton}

Ability to assess the external shape of the nose during the physical examination is largely dependent on knowledge of the topographical points of the face and nose, as well as classic proportions described by Leonardo da Vinci. However, often the practical usefulness of these aesthetic purposes is limited by the anatomy of skeleton and the surrounding skin. 
The examination is performed in an orderly manner, in caudal direction (Elsahy, 2000). The surgeon evaluates the location and slope of the frontonasal and nasolabial angles. Frontonasal angle can be improperly shifted cranially or caudally, so the nose is elongated or abnormally short. Too mild frontonasal angle is an indication for resection of bone or longitudinal muscle. If too sharp, it forms a clearly visible vertex of the frontonasal angle, which is an indication for the cartilage graft (Figure 17).

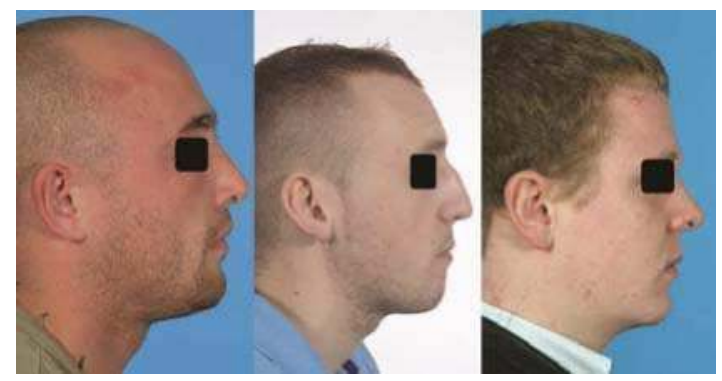

Fig. 17. Frontonasal angle; a) obtuse, b) normal, c) acute

Nasal bones are examined for symmetry, the occurrence of irregularities and their length. It is important to determine the ratio between the width of the base of the nose and the length of the bridge (Elsahy, 2000). Simultaneously, visual illusion must be taken into account on the inverse relation between the projection of the nasal bridge and the size of the nasal base (Figure 18). The aim is to select the optimum reduction of osseous pyramid or augmentation of the area.

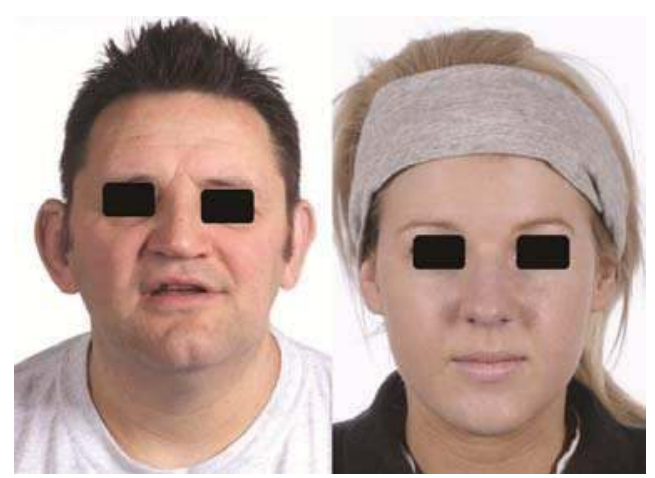

Fig. 18. Two noses with the similar length and a) wider b) narrower nasal base - visual illusion of the shorter nose in the picture on the left because of the wider nasal base

The surgeon assesses the lateral cartilages in terms of their symmetry and strength. Lateral nasal cartilages are compared with nasal bones and nasal septum cartilage in order to assess their supportive function. For shorter nasal bone, the weaker support of the nasal valve is experienced. In these cases, only conserving hump removal can be performed in order to avoid the collapse of the lateral cartilages (Gunter et al., 2007).

Assessment of stiffness and support to the lower third of the nose is of crucial importance in the multivariate analysis of the nose. In order to assess valve pathology, the patient is asked 
to breathe intensively. The surgeon observes collapsing or asymmetry in the nasal side walls, the distortion of the columella, protrusion of the caudal part of nasal septum or collapse of the alae. Reliable screening test (evaluation of 'reversing mechanism') to denote the support of mobile bottom third of the nose consists of strong pressing of the nasal tip to the upper lip using your finger tip, and subsequent sudden release. Then the ability of the nasal tip return to baseline is observed (Krzeski, 2005).

Nasal base is a more complex structure compared to the simple structure of the osseous and upper cartilaginous vaults. In addition, the skin in the caudal pole of the nose is thicker and thus less adapted to surgical manipulation.

Supratip area is evaluated in terms of its position (height, width and recession) and symmetry. In normal conditions it should be flat.

The nasal tip is examined in terms of its projection, rotation, symmetry and position of defining points. Identification of poorly defined tip, a low point of greatest projection tip, convex supratip area, infratip lobule lying above the point of greatest projection helps in planning a thorough correction of this complex anatomical structure.

Nasal alae are examined in terms of width, retraction, and place of connection to columella. The abnormal curvature or asymmetry can be observed. Visual inspections on a peaceful and forceful breathing allow to depict collapse, flaccidity of the cartilage or narrowing of the nasal valve. Palpation determines their size, mobility, shape and strength. The increased distance between the domes of the alae is an indication for correcting the forked nasal tip (Elsahy, 2000).

Infratip lobule is assessed in terms of shape, harmony with the surrounding structures, width, thickness and the ratio of the nasal length and the nasal base width.

Nostrils are assessed in terms of shape and symmetry (Figure 19). At the same time, shape of columella is observed. Examination of columellar width and length allows to assess its supportive function. In case of too short medial crura of alae, there is a need of longitudinal, narrow cartilage graft. Correction of excessive tip projection may include reducing the width and length of too long or widening medial crura of nasal alae (Gunter et al., 2007).

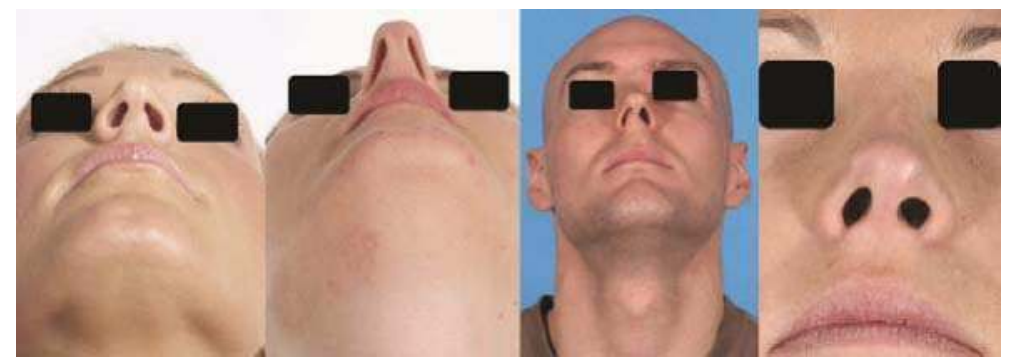

Fig. 19. Shape of nostrils: a) symmetrical teardrop-shaped, b) symmetrical narrow, c) asymmetrical in width, d) asymmetrical in length

The nasolabial angle is examined by observation. Excessively acute angle (less than 80 degrees) indicates a drooping nasal tip - on the assumption that there is no evidence of 'hanging columella' (Figure 20). Excessively acute or retracted nasolabial angle is an indication for tip reduction or transplantation thickening grafts (called plumping grafts) at intersection of columella and the upper lip (subnasale). Excessively obtuse angle is more challenging to repair and requires usually cartilage or bone graft (Krzeski, 2005). 

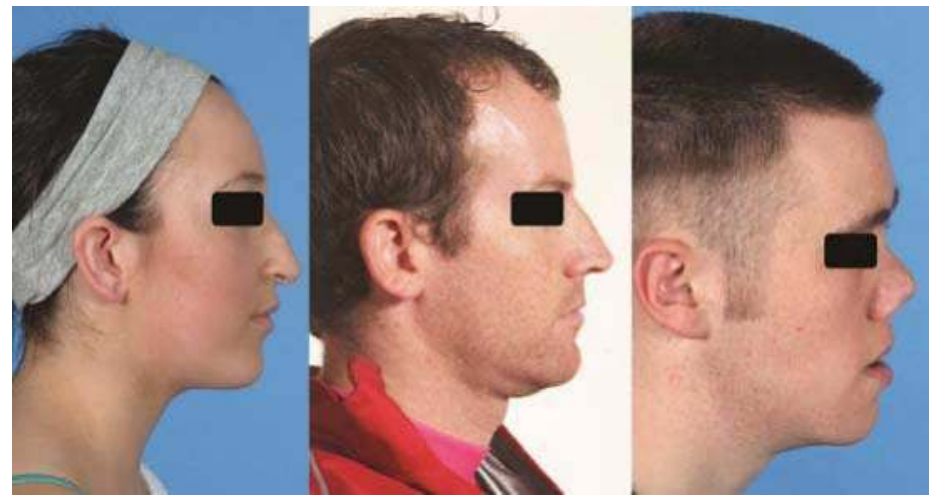

Fig. 20. Nasolabial angle; a) excessively acute, b) normal, c) excessively obtuse

Septal cartilage is examined from the outside in terms of its relation to columella. Palpation is performed with thumb and index finger, allowing to check twisted caudal edge of nasal septum, hypertrophic cartilage septum, caudal distortion of the medial alar crura and columella or structural abnormalities of the anterior nasal spine (Figure 21).

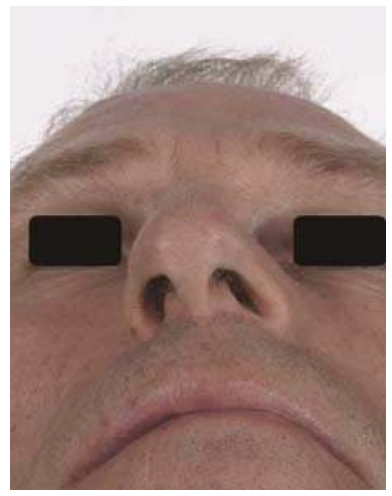

Fig. 21. Distortion of the nasal septum from the midline
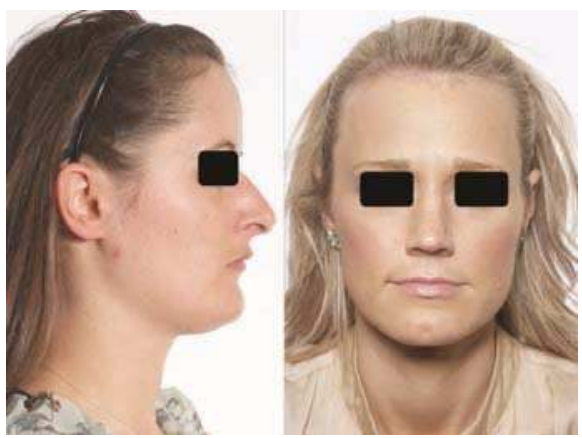

Fig. 22. a) Hanging columella, b) recessed columella 
Excessively protruding columella in the lower pole of the nose, or too big alae, resulting in excessive outline of 'gull in flight', are called 'hanging columella' (Figure 22). In contrast, a flat line is called recessed columella. An important point in nasal analysis is differentiating alar retraction from a hanging columella.

The infratip lobule should be localized below the top of the alar domes. Uncommonly observed 'hanging infratip lobule' occurs when infratip lobule has a too low position relative to the junction of alae.

\subsection{Examination of the internal nose}

Intranasal examination allows to assess the functionality of the nose. It is performed using a speculum, after applying vascular contractile factor in the aerosol (oxymetazoline). The following structures can be assessed: mucosa, nasal turbinates, nasal septum, nasal valve, valve of nasal cavity and nasal ala. The review of the nasal duct confirms nasal patency or indicates the anatomical abnormalities (Tebbetts, 2008).

Assessment of the appearance of mucosa is in consideration of the appearance, atrophic changes, oedema, presence of polyps and anatomical abnormalities in the lateral wall of the nasal cavity.

Nasal turbinates are assessed visually in terms of their size, shape and appearance. Their hypertrophy is common in people with allergies. Because of their functions, the conserving resection should be carried out and only when absolutely necessary (Krzeski, 2004).

Septum is examined to assess its size, shape, coverage of the mucosa, perforation or adhesions. Curvature of the nasal septum posterior from the nasal valve can be detected. Excessive size of septum is noted for its potential use as a graft. Identification of high distortion of the septum (anterior edge) is important to avoid formation of high curvature of the septum after removal of the nasal hump. It is important to detect the curved horizontal cribriform plate because it can cause impaired nasal patency after the lateral osteotomy (Mathes \& Hentz, 2006).

Nasal valve can be easily visualized due to its relatively superficial location. Angle of the valve is formed by the nasal septum and lateral nasal cartilage, amounting normally from 10 to 15 degrees (Trenite, 2005). In order to evaluate the nasal valve, the patient's head is tilted back and the surgeon uses a point light source to observe the presence of a narrow valve, or its collapse on inhalation.

Alae are examined to detect the protrusion of their lateral crura to the vestibule and to assess their support function of the lateral nasal wall.

\section{Photographic documentation}

Pre- and postoperative photographic documentation is a valuable addition to the daily practice of surgeon involved in the rhinoplasty for the following purposes: diagnostic, teaching, self-criticism, as well as medico-legal. Surgeon should obtain written consent from the patient for the photographs to be taken.

\subsection{Two-dimensional photographs}

Routine photographic documentation on a preoperative visit involves an additional 2 minutes, and provides invaluable information on the nasal shape. 
Quality images are obtained using the $105 \mathrm{~mm}$ portrait lens (Mathes \& Hentz, 2006). Patient should be placed approximately $150 \mathrm{~cm}$ from the photographer. Proper lighting provides a set of two lamps directed at an angle of 45 degrees to the camera and the patient. The greatest depth of focus will be provided by optics lens with aperture F-11 to F-22. The background colour should be pale pastel in order to avoid reflection or absorption of an excessive light rays, as well as to be a good complement to the colour of the skin (Tardy, 1997).

Face of the patient should be placed along the Frankfort line. From the front, head and neck should be included. From the side profile, ear and nose should be taken into account. In order to standardize the oblique profile, the lateral border of the face should be adjusted with the tip of the nose. From the bottom, tip of the nose should be positioned between the eyebrows. The patient is placed no closer than $50 \mathrm{~cm}$ from the background in order to avoid the shadows.

Normally, six photographs are carried out in the following projections: front (lens at eye level), lateral (left and right profile - invisible opposite eyebrow), oblique (left and right) and the base of the nose (Figure 23).

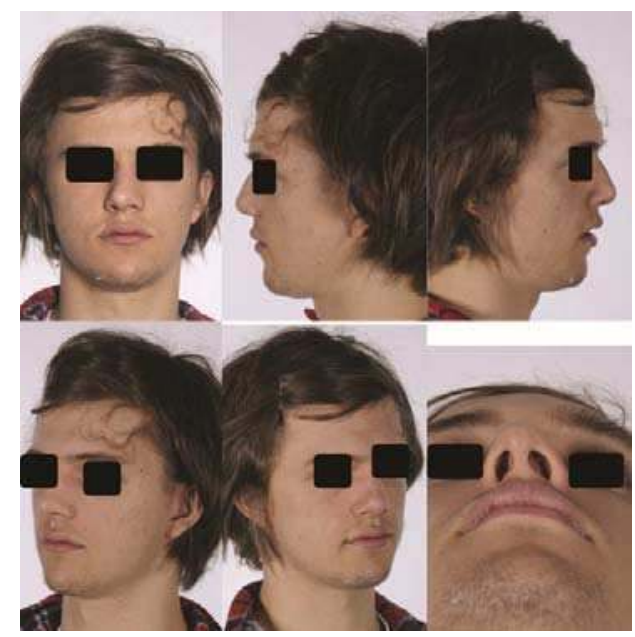

Fig. 23. Routine photographic documentation of the patient prior to rhinoplasty

\subsection{Three-dimensional imaging}

Three-dimensional imaging method allows to determine the shape of a three-dimensional object (e.g. face). Scanning requires set of devices, which transform the image from analog to digital form and include: an optical system, digital communication network and computer with the calculation program. Image captured by an optical system is subjected to computer analysis in the process of digitization. Body surface area is written in the form of points with coordinates in three dimensions. Points are combined with one another to form triangles on poligonisation. The collection of all triangles in a three-dimensional model forms the surface of analysed part of the body, which contains information concerning its actual size (Figure 24). 3D scanner should be configured to allow an accurate assessment of the linear measurements, proportion, area and volume. 
With three-dimensional imaging all currently designated line parameters can be achieved in a repeatable and precise way, as well as additional spatial data describing the shape of the nose, which could be useful for preoperative planning. The postoperative data allow for accurate long-term follow-up. Three-dimensional imaging can also provide invaluable assistance to the surgeon through the preoperative calculation of the needed degree of resection of the nasal cartilages during surgery. Confirmation of usefulness of this method of preoperative planning, however, requires further study.

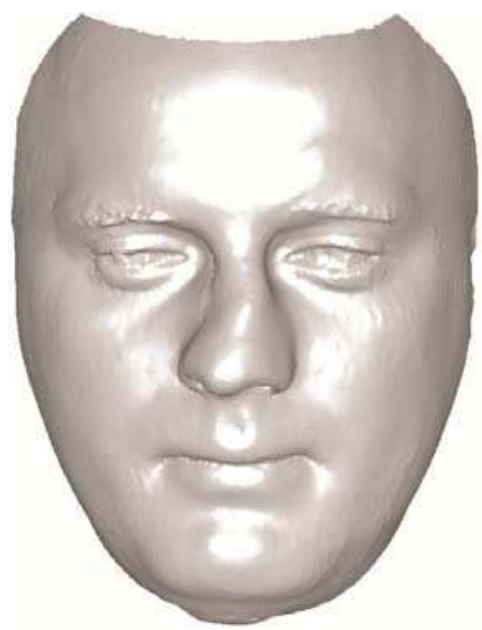

Fig. 24. Three-dimensional model of the face

\section{Additional studies}

Labolatory tests should be performed two weeks prior to rhinoplasty, these may include: blood group, full blood count, ESR, urea + electrolytes, coagulation tests (INR, APTT), HBV antigen, $\mathrm{HCV}$ antibodies.

The following tests may be additionally recommended: WR, total protein, urinalysis, chest X-ray and ECG.

\section{Conclusions}

Proper selection of patients together with detailed preoperative planning, based on accurate knowledge of the nasal anatomy, is essential to achieve good result of rhinoplasty. The relatively fixed points of the nasal surgical anatomy, designated using our protocol of anthropometrical assessment with 3D imaging, can be used for accurate preoperative and intraoperative evaluation. However, ideal proportions of the nose to the whole face act only as guidelines in individualized planning of the surgery.

\section{References}

Alavi, M.; Kalafi, Y.; Dehbozorgi, G.R. \& Javadpour, A. (2011). Body dysmorphic disorder and other psychiatric morbidity in aesthetic rhinoplasty candidates. Journal of 
Plastic, Reconstructive and Aeshtetic Surgery, Vol.64, No.6, (June 2011), pp.738-41, ISSN 1748-6815

Begg, R.J. \& Harkness, M. (1995). A lateral cephalometric analysis of the adult nose. Journal of Oral and Maxillofacial Surgery, Vol.53, No.11, (November 1995), pp.1268-1274, ISSN 0278-2391

Daniel, R.K. \& Farkas, L.G. (1988). Rhinoplasty: image and reality. Clinics in Plastic Surgery, Vol.15, No.1, (January 1988), pp.1-10, ISSN 0094-1298

Daniel, R.K. (2009). Tip Refinement Grafts: The Designer Tip. Aesthetic Surgery Journal, Vol.29, No.6, (November - December 2009), pp.528-537, ISSN 1084-0761

Dziewulski, P.; Dujon, D.; Spyriounis, P.; Griffiths, R.W. \& Shaw J.D. (1995). A retrospective analysis of the results of 218 consecutive rhinoplasties. British Journal of Plastic Surgery, Vol.48, No.7, (October 1995), pp.451-454, ISSN 0007-1226

Elsahy, N. (Ed.). (2000). Plastic and Reconstructive Surgery of the Nose, 1st edn., W.B. Saunders Co., ISBN: 0-7216-7722-3, Philadelphia, USA

Gruber, R.P. \& Peck, G.C. (Eds.). (1993). Rhinoplasty: State of the Art, 1st edn., Mosby, ISBN13 978-0801662775, St. Louis, USA

Gunter, J.P.; Rohrich, R.J. \& Adams, W.P. (Eds.). (2007). Dallas Rhinoplasty - Nasal Surgery by the Masters, 2nd edn., Quality Medical Publishing Inc., ISBN-13 978-1-57626223-8, St. Louis, USA

Krzeski, A. (Ed.). (2004). [Podstawy chirurgii nosa], In Polish. 1st edn., Via Medica, ISBN 8389861-10-0, Gdansk, Poland

Krzeski, A. (Ed.). (2005). [Wyklady z chirurgii nosa]. In Polish. 1st ed., Via Medica, ISBN 8389861-29-1, Gdansk, Poland

Mathes, S.J. \& Hentz, V.R. (Eds.). (2006). Plastic Surgery. Volume II - The Head and Neck, Part 1, 2nd edn., Saunders Elsevier Inc., ISBN-13 978-0-7216-8811-4, Philadelphia, USA

Perrett, D.I.; May, K.A. \& Yoshikawa, S. (1994). Facial shape and judgements of female attractiveness. Nature, Vol.368, No.6468, (March 1994), pp:239-242

Powell, N. \& Humphreys, B. (Eds.). (1984). Proportions of the aesthetic face, 1st edn., Thieme-Stratton, ISBN 0865771170, New York, USA

Rahman, M.; Jefferson, N.; Stewart, D.A.; Oliver, R.; Walsh, W.R. \& Gianoutsos, M.P. (2010). The histology of facial aesthetic subunits: Implications for common nasal reconstructive procedures. Journal of Plastic, Reconstructive and Aesthetic Surgery, Vol.63, No.5, (May 2010), pp.753-756, ISSN 1748-6815

Sevin, A.; Sevin, K.; Erdogan, B.; Adanali, G. \& Deren, O. (2006). A Useful Method for Planning Hump Resection of Deviated Nose. Aesthetic Plastic Surgery, Vol.30, No.4, (August 2006), pp.433-436, ISSN 0364-216X

Simons, R.L. (1982). Nasal tip projection, ptosis, and supratip thickening. ENT - Ear, Nose, \& Throat Journal, Vol.61, No.8, (August 1982), pp.452-455, ISSN 0145-5613

Szychta, P.; Rykala, J. \& Kruk-Jeromin, J. (2010). Assessment of 3D scanner usefulness in aesthetic evaluation of posttraumatic rhinoplasty. The 33rd European Academy of Facial Plastic Surgery Meeting, (September 2010), Belek, Turkey

Talakoub, L. \& Wesley, N.O. (2009). Differences in Perceptions of Beauty and Cosmetic Procedures Performed in Ethnic Patients. Seminars in Cutaneous Medicine and Surgery, Vol.28, No.2, (June 2009), pp.115-129, ISSN 1085-5629 
Tardy, M.E. (Ed.). (1997). Rhinoplasty - The Art and the Science, 1st edn., W.B. Saunders Co., ISBN 0-7216-8755-5, Philadelphia, USA

Tebbetts, J.B. (Ed.). (2008). Primary Rhinoplasty - Redefining the Logic and Techniques, 2nd edn., Mosby Elsevier Inc., ISBN 978-0-323-04111-9, Philadelphia, USA

Tezel, E. \& Durmus, F.N. (2009). A new instrument for achieving a natural nasofrontal angle. Journal of Plastic, Reconstructive \& Aesthetic Surgery, Vol.62, No.12, (December 2009), pp.e617-e619, ISSN 1748-6815

Trenite, G.J.N. (Ed.). (2005). Rhinoplasty: a practical guide to functional and aesthetic surgery, 3rd edn., Kugler Publication, ISBN 90-6299-208-0, Hague, Netherlands 


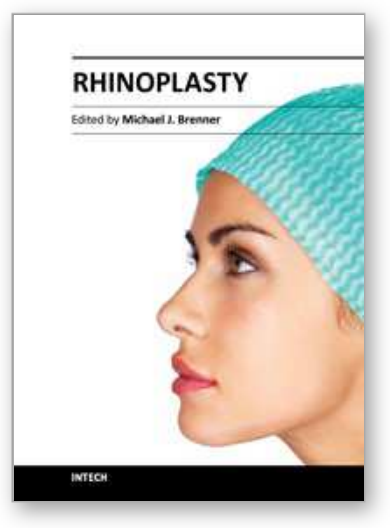

\author{
Rhinoplasty \\ Edited by Dr. Michael Brenner
}

ISBN 978-953-307-849-6

Hard cover, 146 pages

Publisher InTech

Published online 09, December, 2011

Published in print edition December, 2011

Rhinoplasty is one of the defining procedures of plastic and reconstructive surgery. Its roots stem from early efforts in nasal reconstruction to the emergence of modern rhinoplasty. This book describes the latest clinical and research perspectives in rhinoplasty and balances structural correction with aesthetic refinement. With treatises on rhinoplasty from a diverse set of thought leaders from around the world, the collective experience of this books' authors cover cosmetic and reconstructive approaches with a wealth of proven and innovative approaches ranging from minor refinement to major reconstruction. This diversity reflects the inherent complexity of the art and science of rhinoplasty. Discussion of structural approaches is balanced by consideration of judicious resection and refinement. The overarching goal is to instill an understanding of the subtleties of nasal structure and how the natural complexities of nasal anatomy can be adapted to maximize both function and natural appearance.

\title{
How to reference
}

In order to correctly reference this scholarly work, feel free to copy and paste the following:

Pawel Szychta, Ken J. Stewart and Jan Rykala (2011). Preoperative Assessment, Rhinoplasty, Dr. Michael Brenner (Ed.), ISBN: 978-953-307-849-6, InTech, Available from:

http://www.intechopen.com/books/rhinoplasty/preoperative-assessment

\section{INTECH}

open science | open minds

\author{
InTech Europe \\ University Campus STeP Ri \\ Slavka Krautzeka 83/A \\ 51000 Rijeka, Croatia \\ Phone: +385 (51) 770447 \\ Fax: +385 (51) 686166 \\ www.intechopen.com
}

\author{
InTech China \\ Unit 405, Office Block, Hotel Equatorial Shanghai \\ No.65, Yan An Road (West), Shanghai, 200040, China \\ 中国上海市延安西路65号上海国际贵都大饭店办公楼 405 单元 \\ Phone: +86-21-62489820 \\ Fax: $+86-21-62489821$
}


(C) 2011 The Author(s). Licensee IntechOpen. This is an open access article distributed under the terms of the Creative Commons Attribution 3.0 License, which permits unrestricted use, distribution, and reproduction in any medium, provided the original work is properly cited. 